\title{
ANTIBIOTIC RESISTANCE: COMPREHENSION OF THE PROBLEM (REVIEW)
}

\author{
Ivanenko $N$. \\ Donetsk National Medical University (Kropyvnytskyi), Ukraine \\ https://doi.org/10.35339/ic.7.2.60-71
}

\begin{abstract}
Antibiotic resistance (also referred to as drug resistance) is one of the biggest public health challenges of our time. Bacterial infection has become a serious threat to life once again that brings about revision of costly and laborious processes of licensing and regulation of new antibiotics. Understanding the biochemical and genetic basis of resistance is of paramount importance to design strategies to curtail the emergence, spread of resistance, and devise innovative therapeutic approaches to multidrug-resistant organisms. Intrinsic antibiotic mechanisms are normally chromosome-encoded and include non-specific efflux pumps, antibiotic inactivating enzymes, or mechanisms that serve as permeability barriers. The acquired resistance mechanisms are generally obtained by horizontal gene transfer and include plasmid-encoded specific efflux pumps and enzymes that can modify the antibiotic or the target of the antibiotic. These mechanisms pose a more serious threat to human health because of a change in the context of the resistance determinant from chromosomal to plasmid-mediated, resulting in their enhanced expression and dissemination. The collection of resistance genes termed 'resistome' encompasses both intrinsic and acquired resistance genes. Tolerance that can result from mutations and from environmental conditions can occur in a subpopulation of phenotypic variants cells called "persistence", which is a specific type of tolerance. Increasing evidence suggests that tolerance and persistence play a considerable and currently underappreciated role in the recalcitrance and relapse of bacterial infections. The ability of many microbial species to grow as biofilm has further complicated the treatment of infections with conventional antibiotics. The mechanisms of antibiotic tolerance and resistance in biofilms have, in many cases, a genetic basis.

The novel approaches for tackling the antimicrobial resistance crisis must be part of global response to this problem such as phage therapy, antimicrobial peptides (AMPs), bioactive adjuvants, nano-biotechnology, an alternative approaches use gene-specific peptide (PNA), mesenchymal stromal cells, antivirulence therapies, and prophylactic vaccines. The application of specific genome engineering and synthetic biology (SB) methods such as recombineering, clustered regularly interspaced short palindromic repeats (CRISPR), and bacterial cell-cell signaling mechanisms for pathogen targeting are currently essential.

Scientific challenges encompass the discovery molecules with new chemical structures. Economic and scientific obstacles should be overcome by funding researches of advanced drugs and conceptual approaches.

Key words: antibiotic resistance, bacteriophage, biofilm, nanotechnology, new potential
\end{abstract} antibacterial therapy, vaccination.

Antibiotic resistance is one of the biggest public health challenges of our time and can also be referred to as antimicrobial resistance or drug resistance. Each year in the U.S., at least 2.8 million people get an antibiotic-resistant infection, and more than 35,000 people die [1]. Until the early

Corresponding Author:

Nataliia Ivanenko, MD, Assistant to the

Department of Microbiology, Virology and

Immunology, Donetsk National Medical University,

Kropyvnytskyi, Ukraine.

E-mail:natalimarkowskaj@gmail.com 1980s, the pharmaceutical industry developed and introduced many new antibiotics to resolve the resistance issue, and with the passage of time, the pace of antibiotic development staggered, so very few new antibiotics were introduced. It brings about revision of costly and laborious processes of licensing and regulation of new antibiotics, and addresses the economics of antimicrobial drugs (cost of use vs. profit) [2]. Antibiotics are taken for only a short duration for most curable diseases by the patients as compared to drugs for chronic diseases such as heart disease or high-blood pressure, which is the 
reason for companies abandoning the antibiotic area [3].

Antimicrobial resistance (AMR) is a natural phenomenon whereby bacteria evolve in such a way to withstand the action of drugs, making them apparently ineffective. There are four mechanisms through which bacteria become resistance to antibiotics: 1) enzymatic inactivation (for example, beta-lactamase enzymes, which hydrolyze betalactams such as penicillins, cephalosporins); 2) drug extrusion by efflux pumps (proteins, which are able to extrude a wide variety of compounds (including antibiotics) out of the cell; 3) decreased uptake by changes in the outer membrane permeability or by presence of porins; 4) modification of the drug target (these changes impede the binding of the antibiotic and limit its potency) [4]. "Survival of the fittest" is a consequence of an immense genetic plasticity of bacterial pathogens that trigger specific responses that result in mutational adaptations, acquisition of genetic material or alteration of gene expression producing resistance to virtually all antibiotics currently available in clinical practice. Therefore, understanding the biochemical and genetic basis of resistance is of paramount importance to design strategies to curtail the emergence, spread of resistance, and devise innovative therapeutic approaches against multidrug-resistant organisms. In order to provide a comprehensive classification of the antibiotic resistance mechanisms, it is categorized them according to the biochemical and genetic routes involved in resistance. Biochemical reasons: i) modifications of the antimicrobial molecule, ii) prevention to reach the antibiotic target (by decreasing penetration or actively extruding the antimicrobial compound), iii) changes and/or bypass of target sites, and iv) resistance due to global cell adaptive processes. From an evolutionary perspective, bacteria use two major genetic strategies to adapt to the antibiotic "attack", i) mutations in gene(s) often associated with the mechanism of action of the compound, and ii) acquisition of foreign DNA coding for resistance determinants through horizontal gene transfer [5]. Resistance towards antibiotics is acquired by bacteria through either vertical evolution (endogenous) or horizontal evolution (exogenous). Vertical evolution involves the occurrence of a spontaneous mutation within the bacterial genome that confers on the bacterium (and subsequently its progeny) increased resistance to a given compound. Horizontal evolution involves the transfer of a resistance gene from a resistant bacterium to a susceptible bacterium. The mechanisms through which it can occur are conjugation, transduction and transformation [6]. Intrinsic antibiotic mechanisms are normally chromosome-encoded and include non-specific efflux pumps (which is likely evolved as a general response to environmental toxins), antibiotic inactivating enzymes, or mechanisms that serve as permeability barriers. A well-studied example of an intrinsic resistance system is the AcrAB/TolC efflux pump in Escherichia coli and vancomycin resistance in E. coli and other Gramnegative organisms. The acquired resistance mechanisms, on the other hand, are generally obtained by horizontal gene transfer (HGT) and include plasmid-encoded specific efflux pumps (such as TetK and TetL of $S$. aureus) and enzymes that can modify the antibiotic or the target of the antibiotic (mobilization of the chromosomal $\beta$-lactamase gene ampC to a plasmid resulting in its worldwide dissemination). These mechanisms pose a more serious threat to human health because of a change in the context of the resistance determinant from chromosomal to plasmidmediated, resulting in their enhanced expression and dissemination [7].

The collection of resistance genes in a given environment, which is termed the 'resistome', encompasses both intrinsic and acquired resistance genes, as well as proto-resistance genes and silent or cryptic resistance genes. It is thought that many clinically relevant antibiotic resistance genes have their evolutionary origins in environmental microorganisms. Further evidence was provided by a recent functional screen of soil metagenomes, which revealed the presence of environmental antibiotic resistance genes that have $>99 \%$ nucleotide identity to resistance genes in pathogenic isolates [8]. The ability of a whole bacterial population to survive longer treatments with bactericidal antibiotics is denoted as "tolerance". Tolerance that can result from mutations and from environmental conditions is able also to occur in a subpopulation of phenotypic variants cells called "persistence", which specific type of tolerance. Increasing evidence suggests that tolerance and persistence play a considerable and currently underappreciated role in the recalcitrance and relapse of bacterial infections. Tolerance and persistence may facilitate the development of resistance in a more intricate fashion. On the one hand, high levels of persistence or tolerance lead to a higher number of viable cells during antibiotic treatment, which results in an increased statistical probability for the occurrence of resistance- 
conferring mutations. On the other hand, increased persistence, and possibly also tolerance, is pleiotropically linked with increased mutation rates both in growing cells (when antibiotic concentrations are low) and in persisters (when antibiotic concentrations are high). Understanding, detecting, and targeting tolerance and persistence will require joint efforts of microbiologists and clinicians and should eventually lead to reduced therapy failure [9]. Bacteria may adapt to unpredictable disturbances by increasing phenotypic heterogeneity, which can be produced by stochastic noise in gene expression. The variability in gene expression contributes to antibiotic tolerance, due to growth rate dependent killing. A certain frequency of nearly-dormant cells, so called persisters, is naturally produced by stochastic partitioning of proteins after cell division and represents an ancient evolutionary survival strategy, bet-hedging, that can help bacterial populations to survive antibiotic exposure. Phenotypic heterogeneity may thus be an adaptive strategy for the bacteria to cope with unpredictable antibiotic treatments, thereby rendering them inefficient [10].

The ability of many microbial species to grow as biofilm has further complicated the treatment of infections with conventional antibiotics. Indeed, microbial biofilms, that is microbial communities growing attached to abiotic surfaces (medical devices, surgical instruments, industrial pipelines, etc.) and tissues, are known to be an optimal environment to amplify both naturally occurring and induced antibiotic-resistance phenomena. That together with other defense mechanisms significantly increases biofilm antibiotic tolerance [11]. Cells of biofilm that turn to a nongrowing but still active state may quickly acquire tolerance to some agents. The dormant state is also metabolically inactive and nongrowing. To enter the dormant state, however, the cell has implemented protective modifications. Such modifications could include, hypothetically, alteration of membrane lipid and porin composition to reduce permeability, hibernation of ribosomes, inhibition of transcription and replication machinery, and deployment of enzymes that protect against oxidative stress without consuming ATP (e.g., catalase). Examples of stress responses that have been demonstrated in bacterial biofilms include catalase induction upon treatment with hydrogen peroxide, $\beta$-lactamase induction upon treatment with imipenem, and induction of the lipopolysaccharide-modifying $\mathrm{pmr}$ operon upon treatment with colistin. In each of these examples, the induced gene or genes enhance the capacity of the biofilm to tolerate the antimicrobial either by augmenting destruction of the antimicrobial agent or by modifying the cell to make it less susceptible [12]. It is important to acknowledge that, when in a biofilm, pathogenic bacteria employ both tolerance and resistance mechanisms to withstand antimicrobial challenges. It has also become clear that the underlying mechanisms of antibiotic tolerance and resistance in biofilms have, in many cases, a genetic basis. It is generally accepted that the basis for biofilmspecific antibiotic resistance and tolerance is multifactorial, and mechanisms of resistance and tolerance vary depending on the particular antimicrobial agent, the bacterial strain and species, the age and developmental stage of the biofilm, and the biofilm growth conditions [13]. Biofilms are known to control their population density through a cell-to-cell signaling mechanism known as quorum sensing. The benefit of quorum sensing is not limited to controlling population density. In fact, quorum sensing has also been shown to aid the spread of beneficial mutations throughout the biofilm colony, enhance access to nutrients, and contribute to antibiotic tolerance. Biofilms are recalcitrant to antibiotic therapy and a major cause of persistent and recurrent infections by clinically important pathogens worldwide (e.g., Pseudomonas aeruginosa, Escherichia coli, and Staphylococcus aureus). The same mechanisms (mutations, efflux pumps, and antibiotic modifying enzymes) do not appear to be the main cause of biofilm-mediated antibiotic tolerance. The extracellular matrix encasing the cells in a biofilm, also referred to as the Extracellular Polymeric Substance (EPS), is composed of a complex mixture of proteins, lipids, nucleic acids (extracellular-DNA), and polysaccharides. These constituents not only assist in securing the biofilm to the surface, but also trap nutrients, provide structural support, and shield against host immune responses and antimicrobial treatments. In addition, the EPS is also responsible for enabling cell-to-cell communication (quorum sensing), and facilitating the exchange of genetic material through horizontal gene transfer [14].

The antibiotic selection pressure is another factor of modifying the host's microbiota. The host system is subjected to a phenomenon of "selective pressure" when treated with antibiotics during infection. With a greater activity scale, the resistance frequency increases. This results in the resistant species surviving in the host population as compared to the susceptible strain with the harsh effect of the antibiotic. Thus, being a 
reservoir increases the chances of infection spread to a greater extent. Antibiotics like cephalosporins, azithromycin, and fluoroquinolones bring about this effect of "selective pressure" in eukaryotic hosts. A careful and considerate use of antimicrobials is highly recommended for human safety [15]. However, it can be anticipated that antibiotic resistance will continue to develop more rapidly than new agents to treat these infections become available and a better understanding of the molecular, evolutionary and ecological mechanisms governing the spread of antibiotic resistance is needed [16].

The investigation of novel approaches for tackling the antimicrobial resistance crisis must be part of global response to this problem if an untimely reversion to the pre-penicillin era of medicine is to be avoided [17]. The advanced drugs as well as several conceptual approaches are an abundance of new and "old" revisited approaches being studied, which may move us from the end of the antibiotic era towards a new dawn of antibacterial agents. There has been a resurgent interest in the use of lytic bacteriophages to manage bacterial infections [18].

Antibiotics are expected to interfere with aspects of bacterial physiology that can be crucial to phage antibacterial activities, e.g., by interfering with bacterial ribosome functioning. Emphasis here therefore is on documenting the impact of antibiotics on phage-infection pharmacodynamics aspects, i.e., retention of phage ability to negatively impact targeted bacteria despite antibiotic cotreatment. These pharmacodynamic properties include a retention, by bacteria-infecting phages, of both gene expression and antibacterial activity, and also of associated in situ-within treated patients-phage virion production [19]. Now we need to pay more attention to innovatively recruit all phage resources for phage applications. For example, despite better-known lytic DNA phages, we should pay attention to temperate DNA phages, and even RNA phages, for bioplatforms. Given that temperate phages do not rapidly kill the host bacteria, instead actively modifying the properties and behaviors of them, they are likely armed with genetic resources which have evolved to actively manipulate the host's bacterial physiology and may provide clues concerning new antibacterial targets and proteins. As exemplified, was identified a phage protein (Tip) and its new antipathogenic target (PilB), from an unmodified temperate phage that displayed therapeutic efficacy in acute infections caused by $P$. aeruginosa. In that temperate phages' life cycles have focused on establishing a co-flourishing symbiosis with that of their host bacteria, they may be advantageous over lytic phages for reducing the emergence of antibacterial resistance. [19]. Many antibiotics (fluoroquinolones and $\beta$-lactams) can induce the expression of prophage gene products or lead to the excision and propagation of temperate phages activating and spreading hitchhiker prophages. This antibiotic effect may have unexpected outcomes on the virulence of bacterial populations and their resistance to antimicrobial drugs. These unwanted side-effects of antibiotics cast doubt on the suitability of some antimicrobial treatments and may require new strategies to prevent and limit the selection for virulence [18]. The very interaction of phages with the surface of bacterial cells may itself have an additive effect for phage therapy. It has been shown that bacteriophages, by attaching themselves to the bacterial surface at particular sites, could block resistance mechanisms such as an efflux pump or impair the fitness or the virulence factor of a bacterium. This would then make certain bacteria (i.e., P. aeruginosa or K. pneumoniae) more susceptible to traditional antibiotics and facilitate the healing of certain pathologies, such as endocarditis or vascular prosthesis infections [20].

The invaluable advantage of the specificity of phages is also a disadvantage: A suitable phage must be found/screened for an individual patient; a phage mixture could be an alternative in acute situations. The larger a stock collection of purified phages for a certain bacterial species is, the better the chance to find suitable phages [21]. The approach to phage therapy should be doubly effective; success is achieved when phage lyse the target bacterium, but also when bacteria evolve phage resistance because they suffer reduced virulence or increased sensitivity to antibiotics [22].

Bioengineering of phages could dramatically increase their therapeutic potential via a range of mechanisms, including expanded host range, switching host tropism, delivery of exogenous genes, or modification of phage capsids. The research was carried out for the host range of $\mathrm{E}$. coli phage $\mathrm{T} 2$ by incorporating the long tail fiber genes of phage IP008 through homologous recombination. The resulting chimeric phage exhibited the broader host range of phage IP008, while still maintaining the strong lytic activity of phage T2. The engineered virus was capable of reducing biofilm cell counts by $>100$-fold compared to the wild-type phage. The genetic material that can be delivered or inserted into the 
bacterial cell includes dominant sensitive genes to reverse antibiotic resistance, CRISPR/Cas9 sequences to inactivate virulence genes, modified lethal transcription regulators, small regulatory RNAs to silence antibiotic resistance determinants, and even genes that code for proteins capable of increasing the susceptibility to specific antibiotics [23]. Phage engineering technologies allow generating variants with unique properties and helping minimum the features that might hamper the applications of phage for prophylactic and therapeutic applications. All these methods are based on the homologous recombination. Alternatively, engineered phages can be directly generated by transforming the host cells with naked full-length phage genomic DNA containing the desired mutations [24]. Two slightly different medium-term strategies have been developed: the "magistral" phage production, introduced in Belgium recently, and the sustainable large-scale long-term access supply inventory, the latter requiring phage banks with substantial holdings of purified or pre-purified phages, both finally tailor-made flexible medicine. For true emergency applications with less common bacterial strains, compassionate use for individuals in hopeless situations should be possible. It seems crucial to include allowing immediate use of phages newly isolated with minimal regulatory requirements beyond those defining a complete production route. It is an additional advantage that phages and antibiotics are acting synergistically so that overnight phage screenings (phagograms) against patient isolates in parallel with antibiograms can produce results for tailored application [21].

Another approach considers the antimicrobial peptides (AMPs), which are predominantly small cationic peptides with hydrophobic regions containing between 10-100 amino acids, especially arginine residues, which allows them to interact with negatively-charged membranes, causing the direct destabilization of the surface of membranes with pore formation and subsequent cell lysis. Also, they have been described as chemotactic agents, modulating the immune system, and therefore constituting a bridge between innate immunity and adaptive immunity [25]. Although the principal antibacterial activity is attributed to their membrane-lytic mechanism, AMPs have also been demonstrated to function in host immune modulation, often by enhancing protective immunity and suppressing inflammation. They can influence processes like cytokine production, antigen presentation, chemotaxis or wound healing, complementing their bactericidal activity.
For example, the AMP LL-37, found mainly in the granules of neutrophils, is an antisepsis agent that upregulates the production of interleukin- 8 and leads to recruitment of leukocytes to the site of infection. Antimicrobial peptides and proteins with similar characteristics are very promising for the development of potential therapeutics to use against multiple antibiotic-resistant infectious diseases. Aside from the empowering effect in the immune system and their membrane-lytic abilities, AMPs can affect several bacterial processes such as macromolecular synthesis (i.e., RNA, DNA synthesis) protease inhibition, and protein-folding inhibition [26]. The most abundant synergy, including the interactions with human endogenous AMPs, is observed for antibiotics targeting protein biosynthesis, such as aminoglycosides and macrolides. Thus, these antibiotics may enhance the antimicrobial activity of host defensive molecules as well as can be used in combinations with AMP-derived antimicrobial drugs. The ability of AMPs to permeabilize bacterial membranes plays central role in their synergy with other antimicrobial compounds, but also indicates that this ability could be in turn modulated by the second substance in the combination contributing to the combined effect [27]. The diverse array of AMPs acts through different mechanism, and because many AMPs are bactericidal as opposed to bacteriostatic, it is unlikely that bacteria will be able to respond to these AMPs by adopting resistance strategies. Mesenchymal stromal cells (MSC) are adult multipotent progenitor cells, present in a variety of tissues and organs and contribute to healing processes by participating in the inflammatory, proliferative and remodeling phases of tissue repair. The present study is the first to show that equine MSC possess antimicrobial properties by inhibiting the growth of $E$. coli and $S$. aureus, in part by secreting antimicrobial peptides (AMPs) and depolarizing bacterial cell membranes. This antibacterial activity may contribute to the value of MSC as a therapy for chronic cutaneous wounds, where colonization by pathogenic bacteria commonly inhibits normal healing [28]. Accumulating evidence shows that in addition to acting at the cell membrane, AMPs may act on the cell wall, inhibit protein folding or enzyme activity, or act intracellularly in different mechanism of action on gram-negative and gram-positive bacteria. While AMPs should not cause widespread resistance due to their preferential attack on the cell membrane, in cases where specific protein targets are involved, the possibility exists for 
genetic mutations and bacterial resistance. Indeed, the potential clinical use of AMPs has raised the concern that resistance to therapeutic AMPs could be associated with resistance to endogenous host-defense peptides. Current evidence suggests that this is a rare event that can be overcome by subtle structural modifications of an AMP [29].

Many AMPs exhibit direct microbial killing activity and also play an integral role in the innate immune system. These properties make AMPs attractive alternative antimicrobial agents. Furthermore, AMPs are promising candidates to be used as adjuvants in combination with current antibiotics in order to combat antibiotic resistance. Combinations of AMPs and antibiotics are less likely to develop resistance or transmit crossresistance. The further identification and therapeutic development of AMPs and antibiotic-AMP combinations are strongly recommended [30]. Recent studies have shown that in particular cationic AMPs, such as LL-37, piperacillin, buforin II, ceprocin P1, indolicidin, nisin, and magainin II, are remarkably effective in combination with antibiotics like polymyxin E, piperacillin, azithromycin, daptomycin, linezolid, and clarithromycin to enhance antibiotic bioavailability against highly multidrug-resistant gram-negative and methicillinresistant $S$. aureus (MRSA) pathogens. More than to enhance oral bioavailability, the strong membrane permeabilization capacity of AMPs (a novel synthetic cyclolipopeptide analog of polymyxin AMP38) can directly kill even dormant biofilm-forming cells in combination with classical antibiotics. An example demonstrating the efficacy of AMP-antibiotic therapy to remove biofilm is the treatment of Pseudomonas aeruginosa ( $P$. aeuruginosa) with carbapenems [31].

The paradigm entails the use of bioactive adjuvants that augment the antibiotic efficacy of a primary antibiotic against drug-resistant pathogens. The adjuvant may possess weak to no antibacterial activity on its own but is able to either impede antibiotic resistance mechanisms or potentiate antibiotic action. An adjuvant may be an efflux pump inhibitor (EPI) (to prevent the extrusion of drugs), a membrane permeabilizer (to increase the number of molecules that penetrate the membrane), or an enzyme inhibitor (to prevent the degradation of drugs before they reach their targets). Clavulanic acid by itself possesses poor intrinsic activity against pathogens, but it efficiently inhibits widespread $\beta$-lactamases such as many types of the extended-spectrum $\beta$-lactamase (ESBL) family. The adjuvant aspergillomarasmine A (AMA) was recently discovered to resuscitate the biocidal activity of the carbapenem drug meropenem against metallo$\beta$-lactamase-producing organisms [32]. One such approach is the use of adjuvants capable of revitalizing the activity of current existing antibiotics from resistant pathogens. Recently were reported a series of tobramycin (TOB)-based hybrid adjuvants that were able to potentiate multiple classes of legacy antibiotics (fluoroquinolones (moxifloxacin and ciprofloxacin), tetracyclines (minocycline), or rifamycin (rifampicin) against various multidrug-resistant (MDR) Gramnegative bacteria (GNB) [33].

However, despite the multiple beneficial properties of AMPs, they present some disadvantages such as: (I) degradation by proteases, both in the bloodstream and in the gastrointestinal system; (II their union with others proteins, which leads to their inactivation; (III) low metabolic stability and oral absorption; (IV) rapid excretion through kidneys and liver; (V) high toxicity and immunogenicity; and (VI) high production costs. For these reasons, their use for in vivo applications has not been fully satisfactory and only a few of them were explored in clinical trials. One of the main goals of nanotechnology is the design of nano-carriers, promising biomaterials that could increase therapy efficacy, minimize side-effects, and offer a controlled pharmacokinetic profile. Diverse types of organic nano-materials, including polymers (natural polymers such as DNA, cellulose, or chitosan, and many others, such as poly(lactide-co-glycolide) (PLGA) or PEG, are synthetic), liposomes, hydrogels, self-assembly systems formed by surfactants, (block co)polymers, and polar lipids polymer (micro)gels are utilized for study. A wide range of inorganic nanoparticles/ nano-materials, each offering system-specific opportunities, have been explored as delivery systems not only in the transport of peptides, but also for gene therapy, cancer treatment, and drug delivery such as gold nanoparticles (AuNPs), silver nanoparticles (AgNPs), carbon nanotubes (CNTs) [34], $\mathrm{ZnO}, \mathrm{CuO}$ and $\mathrm{Fe} 2 \mathrm{O} 3$ [18]. It has been reported that peptides in nanostructures presented lower cytotoxicity, reduced degradation and increased efficiency at the desired target [35]. Nano-materials can be defined as the materials that have at least one dimension in the nano range (1-100 nm), whereas nanoparticles (NPs) are particles with at least one dimension in the nano range and can be as small as $0.2 \mathrm{~nm}$. There are five features of nano-materials that make them a possible alternative to antibiotics. First, nano- 
materials can easily penetrate the bacterial cell membrane and damage its structure, resulting in bacterial cell death. Second, suggested mechanisms of antibacterial activity of nano-materials are similar to the action of antibiotics, including reactive oxygen species (ROS)-mediated oxidative stress, cell membrane disruption, intracellular protein synthesis inhibition, and leakage of intracellular components. ROS mainly include superoxide $(\mathrm{O} 2 \bullet-)$, hydroxyl radical $(\bullet \mathrm{OH})$, singlet oxygen (1O2), and hydrogen peroxide $(\mathrm{H} 2 \mathrm{O} 2)$, and generation of ROS by nanomaterials is regarded as the main reason for nanomaterial-mediated antibacterial activity. Third, various nano-materials can act as antibiotic drug carriers to help effectively administer antibiotics to their target locations by reducing the probable adverse effects of antibiotics. Fourth, the retention power of nano-materials in the body is much more than antibiotics, which could be favorable for long-term therapeutic effects. Finally, nano-materials can be functionalized according to their target and purpose of use as they can be effective against bacterial cells without being toxic against mammalian cells [36]. Antibiotics encapsulated in NPs have a large capacity to be replaced with antibiotics in free form. There are numerous benefits in nanoencapsulation strategy such as very-small size, a big surface-zone-to-mass ratio, high loading capacity and high reactivity, protection of antibiotics against physical inactivation, improvement in antibiotics pharmacokinetics, facilitation of the antibiotic release in infection area and reducing the required dose of drug. The effect of aptamerconjugated nanoparticles on the biofilm formation has been also evaluated. For example, singlewalled carbon nanotubes (SWNTs) with significant antimicrobial activity were utilized as a nanomaterial to detect Pseudomonas aeruginosa as well as it could increase the toxic effect of antimicrobial agents on bacterial biofilms [37]. Nanotechnology has vast opportunity to command and modify molecular structures at nano-scale to attain specific target action. Nanobullet targeting is advantageous over conventional systems as they enhance therapeutic capacity by preventing microbial resistance. Furthermore, nano-targeting often prevents frequent drug intake and reduces side effects. Therefore, nano-science enhances patient compliance through protecting natural microbiome. NPs have the capability to overcome drug resistance due to their multifunctionality, as bacteria cannot develop multiple gene mutations simultaneously [38].
Other approaches include use of genomics to find out new bacterial targets and optimization of newer approaches that target bacterial pathogens while exerting selection for reduced pathogenesis, if bacteria evolve resistance to therapeutic intervention [39]. An alternative approach could be to use gene-specific oligonucleotides, such as peptide nucleic acid (PNA) oligomers, that can specifically target any single pathogen and PNA as a nucleic acid mimic. This approach broadens the range of potential targets to any gene with a known sequence in any bacterium, and could significantly reduce the time required to discover new antimicrobials or their redesign, if resistance arises. Antisense oligonucleotides, used to inhibit the synthesis of proteins essential for bacteria to sustain life, may be helpful in the fight against bacterial infections. The peptide nucleic acid (PNA) molecule combines the properties of both peptides and nucleic acids. In the last two decades, many mRNA encoding essential genes in clinically pathogenic bacteria have been validated as possible targets for antisense PNA.

The PNA sequences can be used as a potential structure with antibacterial properties in gene expression. The first reported antibacterial PNA targeted the mRNA transcript of E. coli acpP gene that encodes the acyl carrier protein, a protein crucial in fatty acid biosynthesis. To improve the antimicrobial activity, PNA have also been used in combination with antibiotics. These included aminoglycosides, penicillins, polymyxins, rifamycins, sulfonamides, and trimethoprim. Other essential biological processes that have been disrupted by antisense PNA, in both gramnegative and gram-positive bacteria, are DNA transcription and replication. Respectively, the rpoD gene encoding RNA polymerase and gyrA encoding DNA gyrase were targeted by PNA in several pathogens including $S$. pyogenes, S. aureus, S. Typhimurium and Shigella flexneri. Nevertheless, the use of PNA as an antibiotic is not foreseen in the near future due to crucial limitations. The main drawback precluding the use of PNA as an antimicrobial is its lack of uptake by bacterial cells [40].

The acronym ESKAPE includes six nosocomial pathogens that exhibit multidrug resistance and virulence: Enterococcus faecium, Staphylococcus aureus, Klebsiella pneumoniae, Acinetobacter baumannii, Pseudomonas aeruginosa, and Enterobacter spp. Persistent use of antibiotics has provoked the emergence of multidrug resistant (MDR) and extensively drug resistant (XDR) bacteria, which render even 
the most effective drugs ineffective. Alternative therapies such as use of antibiotics in combination or with adjuvants, bacteriophages, antimicrobial peptides, nanoparticles, and photodynamic light therapy are widely reported. Some of the commonly described limitations of these therapies include stability and toxicity of the therapeutic agent, its targeted delivery at the site of infection, or immune response developed by the host against the therapeutic agent. Ongoing research has therefore led to further develop or modify these novel therapeutic agents or therapies so as to surmount the limitations as well as to overcome the barriers of bacterial resistance [41]. Consequently, the discovery and development of biofilm eradication agents as comprehensive and provide an overview of biofilm remediation strategies, focusing primarily on the most promising biofilm eradication agents and approaches [42]. Proteases secreted by equine mesenchymal stromal cells (MSC) are responsible for the degradation of proteins in MRSA biofilms. In addition to MRSA, was demonstrated that mature biofilms of $\mathrm{P}$. aeruginosa, $\mathrm{S}$. aureus, and S. epidermidis were reduced by MSC conditioned media (CM) as well. Furthermore, was demonstrated that equine MSC secrete cysteine proteases that destabilize MRSA biofilms, thereby increasing the efficacy of antibiotics that were previously tolerated by the biofilms [43]. However, the problem for Gram-negative bacteria is conceivably graver than Gram-positive pathogens due to these being more commonly multidrug-resistant (MDR). Thus, only six of these (ceftolozane-tazobactam, ceftaroline fosamil, ceftazidime-avibactam, meropenemvaborbactam, delafloxacin and secnidazole) have been developed and found effective in the treatment of drug-resistant Gram-negative bacterial infections. The most widely studied hybrid compounds contain the fluoroquinolone class of antibiotic linked to another antibacterial agent. Since the last few years, many antibiotic hybrids have entered trials, but only a few have been reported to progress to clinical trials [44].

An alternative to killing bacteria or stopping their growth, is to search for drugs that disarm bacteria. This idea focuses on developing drugs that inhibit bacterial virulence rather than bacterial viability.

On the other hand, development of antivirulence therapies presents its own unique challenges. We can no longer use established screening systems that identify compounds that kill or inhibit growth of bacteria and minimal inhibitory concentration measures are obsolete in this scenario. Given that virulence mechanisms vary from one bacterium to another, antivirulence drugs are likely to have a narrow spectrum of activity. Their success in the clinic may well depend on development of real time diagnostics that identify the causative organism and enable therapy personalized to the infectious agent. However, aside from antibodies that inactivate specific bacterial toxins, none of these compounds with new mechanisms of actions has yet reached the clinic. So it remains to be seen whether all or some of these antivirulence approaches will live up to expectations [45]. Anti-virulence may offer a new wave of potential antibacterial therapeutics in the future, which drugs that will presumably have longer periods of clinical usefulness, compared to antibiotics. From the viewpoint of the development of virulence inhibitors, inhibition of quorum sensing (QS) is a promising route because various important features in bacterial physiology and virulence are mediated by QSdependent gene expression (e.g., production of toxic shock syndrome toxin in Staphylococcus aureus, elastase in $P$. aeruginosa, protease in $V$. cholerae; activity of bacterial secretion systems (e.g., Salmonella species) and efflux pumps (e.g., P. aeruginosa, Escherichia coli), biofilm-production (e.g., P. aeruginosa, Acinetobacter baumannii, Stenotrophomonas maltophilia); induction of bacterial competence (e.g., Streptococcus pneumoniae), motility (e.g., P. aeruginosa), adhesion (e.g., E. coli, Klebsiella pneumoniae) and pigment-production (e.g., Chromobacterium violaceum, Serratia marcescens, $P$. aeruginosa) [46].

It is less well known that prophylactic vaccines also are highly effective and valuable tools to fight antibiotic resistance. Disease prevention by vaccination lowers antibiotic use and reduces resistance. Both Haemophilus influenzae type B (Hib) and pneumococcal conjugate vaccines are instructive examples and success stories having demonstrated their effectiveness in reducing antibiotic use and reducing resistance. Vaccines that are currently in clinical development differ from these earlier vaccines in that they are designed to address more comprehensively the complex pathophysiology of $\mathrm{S}$. aureus infection by eliciting antibodies that target multiple virulence factors. Furthermore, the antibody responses elicited are functional and either kill the bacteria or neutralize the virulence factors [47]. While resistance is a predictable outcome of antibiotic use, resistance to vaccines 
is an extremely rare event. One possibility is that vaccines typically restrict the ability of the pathogen to establish a foothold in the host, by conferring immunity before infection with the pathogen. Thus, vaccines reduce the chance that some bacteria mutate and become resistant, and also reducing the chance that these resistant genes are spread to other bacteria. The risk of a rare mutation occurring that allows the pathogen to resist the effect of vaccination is thus lower than it is with antibiotic treatment. In total, this overuse and/or misuse of antibiotics exerts selective pressure on bacteria overall, which facilitates the rise and transmission of resistance [48].

The advent of new bacterial genome engineering and synthetic biology (SB) tools is providing promising diagnostic and treatment plans to monitor and treat widespread recalcitrant bacterial infections. Key advances in genetic engineering approaches can successfully aid in targeting and editing pathogenic bacterial genomes for understanding and mitigating drug resistance mechanisms. The application of specific genome engineering and SB methods such as recombineering, clustered regularly interspaced short palindromic repeats (CRISPR), and bacterial cell-cell signaling mechanisms for pathogen targeting are currently essential. Apart from gene insertions, deletions or mutations for modification of the genome using engineering tools, one of the main goals of SB is to build and integrate gene circuits which process signals within a living cell for a desired output. The mobile group II introns are ribozymes that can insert into specific targets by the process of retrohoming. Using predictive algorithms, the intron RNA can be re-designed to form a 'targetron', such that a target DNA site of choice can be edited. This method has been adapted to a number of pathogenic strains for understanding mechanisms of virulence [49].

In any case, the war against antimicrobial resistance is not lost. We must continue the fight, which requires a better knowledge of the mechanisms of action of anti-infectious agents and concomitantly the mechanisms of resistance of infectious agents, a better and fairer use of antibiotics. We need "real" new molecules, i.e., with new chemical structures. Indeed, on 19 August 2019, the FDA approved the marketing of a brand-new antibiotic: Lefamulin, for the treatment of community-acquired bacterialpneumonia (CABP). Lefamulin is the first antibiotic with a novel mechanism of action approved by the FDA in nearly 20 years. Lefamulin belongs to the family of pleuromutilins, which targets a different protein synthesis binding site than older antibiotics (i.e., it inhibits of protein synthesis by binding to the peptidyl transferase center of the $50 \mathrm{~S}$ bacterial ribosome, thus preventing the binding of transfer RNA for peptide transfer). Lefamulin represents a great hope in the search for real new antibacterials. We must therefore continue to search for new antibacterial molecules and synthetic or natural molecules to feed the pipeline, because we will never stop needing new antibacterials [50]. The essential questions are now focused on the biology of the drug, with the PK/PD (pharmacokinetic (PK)/pharmacodynamic (PD) parameters) predictions of outcome in animal tests along with data from human volunteers and patients to act as surrogates for microbiological and clinical efficacy [51].

Conclusions. The development of new antibiotics is impeded by cut in financing by pharmaceutical companies the discovery of new antibiotic drugs. The reasons for these limitations in funding are both economic and scientific issues. Antibiotic resistance will continue to develop faster than appearance new antibiotics or validity the existent drugs available for treatment current infections. Bacteria elaborate new adaptive mechanisms frequently that can protect them against antibiotics. All of these need to investigate new antimicrobial strategies that become of vital importance. Manipulating the new approaches based on insights into metabolic and genetic properties of bacterial cell can enhance the possibility to withstand the proceeding antibiotic resistance. The goal in the future will be to explore the potential of some antimicrobial therapies and strategies to boost antibiotic applying. Advances genetic engineering approaches can be beneficial in targeting and editing pathogenic bacterial genomes for understanding and mitigating drug resistance mechanisms. Therefore, developing the new antibacterial, synthetic or natural molecules to feed the pipeline is a difficult ongoing question.

Conflict of interests. There is no conflict of interests.

\section{References}

1. Centers for Disease Control and Prevention. Antibiotic/Antimicrobial Resistance (AR/AMR). (2020). Retrieved from https://www.cdc.gov/drugresistance/index.html 
2. Aslam, B., Wang, W, Arshad, M.I., Khurshid, M., Muzammil, S., Rasool, M.H., Nisar, M.A., Alvi, R.F., Aslam, M.A., Qamar, M.U., Salamat, M.K.F., Baloch, Z. (2018). Antibiotic resistance: a rundown of a global crisis. Infect Drug Resist., 11: 1645-1658. doi: 10.2147/IDR.S173867.

3. Martens, E., Demain, A. (2017). The antibiotic resistance crisis, with a focus on the United States. J Antibiot, 70(5):520-526. doi: 10.1038/ja.2017.30.

4. Annunziato, G. (2019). Strategies to Overcome Antimicrobial Resistance (AMR) Making Use of Non-Essential Target Inhibitors: A Review. Int J Mol Sci., 20(23): 5844. doi: 10.3390/ijms20235844.

5. Munita, J. M., Arias, C. A. (2016). Mechanisms of Antibiotic Resistance. Microbiol Spectr, 4(2). doi: 10.1128/microbiolspec.VMBF-0016-2015.

6. Laws, M., Shaaban, A., Rahman, K.M. (2019). Antibiotic resistance breakers: current approaches and future directions. FEMS Microbiol Rev, 43(5):490-516. doi: 10.1093/femsre/fuz014.

7. Peterson, E., Kaur, P. (2018). Antibiotic Resistance Mechanisms in Bacteria: Relationships Between Resistance Determinants of Antibiotic Producers, Environmental Bacteria, and Clinical Pathogens. Front Microbiol., 9: 2928. doi: 10.3389/fmicb.2018.02928.

8. Crofts, T.S., Gasparrini, A.J., Dantas, G. (2017). Next-generation approaches to understand and combat the antibiotic resistome. Nat Rev Microbiol., 15(7):422-434. doi: 10.1038/nrmicro.2017.28.

9. Windels, E. M., Michiels, J. E., Van den Bergh, B., Fauvart, M., Michiels, J. (2019). Antibiotics: Combatting Tolerance To Stop Resistance. mBio, 10(5). pii: e02095-19. doi: 10.1128/mBio.02095-19.

10. Roemhild, R., Schulenburg, H. (2019). Evolutionary ecology meets the antibiotic crisis: Can we control pathogen adaptation through sequential therapy? Evol Med Public Health. 2019(1):37-45. doi: 10.1093/emph/eoz008.

11. Francolini, I., Piozzi, A. (2019). Polymeric Systems as Antimicrobial or Antifouling Agents. Int J Mol Sci., 20(19):4866. doi: 10.3390/ijms20194866.

12. Stewart, P.S. (2015). Antimicrobial Tolerance in Biofilms. Microbiol Spectr.,3(3):10.1128/ microbiolspec.MB-0010-2014.doi: 10.1128/microbiolspec.MB-0010-2014.

13. Hall, C.W., Mah, T.F. (2017). Molecular mechanisms of biofilm-based antibiotic resistance and tolerance in pathogenic bacteria. FEMS Microbiol Rev., 41(3):276-301. doi: 10.1093/femsre/fux010.

14. Verderosa, A.D., Totsika, M., Fairfull-Smith, K.E. (2019). Bacterial Biofilm Eradication Agents: A Current Review. Front Chem., 7:824. doi: 10.3389/fchem.2019.00824.

15. Ray, S., Das, S., Suar, M. (2017). Molecular Mechanism of Drug Resistance. Drug Resistance in Bacteria, Fungi, Malaria, and Cancer, 47-110. doi: 10.1007/978-3-319-48683-3_3.

16. Medina, E., Pieper, D. H. (2016). Tackling Threats and Future Problems of Multidrug-Resistant Bacteria. Curr Top Microbiol Immunol., 398:3-33. doi: 10.1007/82_2016_492.

17. Laws, M., Shaaban, A., Rahman, K.M. (2019). Antibiotic resistance breakers: current approaches and future directions. FEMS Microbiol Rev, 43(5):490-516. doi: 10.1093/femsre/fuz014.

18. Tillotson, G.S., Theriault, N. (2013). New and alternative approaches to tackling antibiotic resistance. F1000Prime Rep., 5:51. doi: 10.12703/P5-51.

19. Abedon, S. T. (2019). Phage-Antibiotic Combination Treatments: Antagonistic Impacts of Antibiotics on the Pharmacodynamics of Phage Therapy? Antibiotics (Basel), 8(4). pii: E182. doi: 10.3390/ antibiotics 8040182 .

20. Patey, O., McCallin, S., Mazure, H., Liddle, M., Smithyman, A., Dublanchet, A. (2018). Clinical Indications and Compassionate Use of Phage Therapy: Personal Experience and Literature Review with a Focus on Osteoarticular Infections. Viruses, 11(1):18. doi: 10.3390/v11010018.

21. Rohde, C., Wittmann, J., Kutter, E. (2018). Bacteriophages: A therapy concept against multidrug-resistant bacteria. Surg Infect (Larchmt), 19 (8), 737-744. doi: 10.1089/sur.2018.184.

22. Kaitlyn, E. Kortright, Benjamin, K. Chan, Jonathan, L. Koff, Paul, E. Turner. (2019). Phage Therapy: A Renewed Approach to Combat Antibiotic-Resistant Bacteria. Cell Host Microbe, 25 (2), 219 232. doi: 10.1016/j.chom.2019.01.014.

23. Gordillo Altamirano, F.L., Barr, J.J. (2019). Phage Therapy in the Postantibiotic Era. Clin Microbiol Rev., 32(2):e00066-18. doi: 10.1128/CMR.00066-18.

24. Chen, Y., Batra, H., Dong, J., Chen, C., Rao, V.B., Tao, P. (2019). Genetic Engineering of Bacteriophages Against Infectious Diseases. Front Microbiol., 10:954. doi: 10.3389/fmicb.2019.00954.

25. Martin-Serrano A, Gomez R, Ortega P, de la Mata FJ. (2019). Nanosystems as Vehicles for the Delivery of Antimicrobial Peptides (AMPs). Pharmaceutics, 11(9):448. doi: 10.3390/ pharmaceutics11090448. 
26. Monserrat-Martinez, A., Gambin, Y., Sierecki, E. (2019). Thinking Outside the Bug: Molecular Targets and Strategies to Overcome Antibiotic Resistance. Int J Mol Sci., 20(6):1255. doi: 10.3390/ ijms20061255.

27. Zharkova, M.S., Orlov, D.S., Golubeva, O.Y., Chakchir, O,B., Eliseev, I.E., Grinchuk, T.M., Shamova, O.V. (2019). Application of Antimicrobial Peptides of the Innate Immune System in Combination With Conventional Antibiotics-A Novel Way to Combat Antibiotic Resistance? Front Cell Infect Microbiol, 9:128. doi: 10.3389/fcimb.2019.00128.

28. Harman, R.M., Yang, S., He M. K., Van de Walle, G.R. (2017). Antimicrobial peptides secreted by equine mesenchymal stromal cells inhibit the growth of bacteria commonly found in skin wounds. Stem Cell Res Ther., 8: 157. doi: 10.1186/s13287-017-0610-6.

29. B. Bechinger, S.-U. Gorr. (2016). Antimicrobial Peptides: Mechanisms of Action and Resistance. J Dent Res, 96 (3), 254-260. doi: 10.1177/0022034516679973.

30. Lewies, A., Du Plessis, L.H., Wentzel, J.F. (2019). Antimicrobial Peptides: the Achilles' Heel of Antibiotic Resistance? Probiotics Antimicrob Proteins., 11(2):370-381. doi: 10.1007/s12602-018-9465-0.

31. Pizzolato-Cezar, L.R., Okuda-Shinagawa, N.M., Machini, M.T. (2019). Combinatory Therapy Antimicrobial Peptide-Antibiotic to Minimize the Ongoing Rise of Resistance. Front Microbiol., 10:1703. doi: 10.3389 /fmicb.2019.01703.

32. Domalaon, R., Idowu, T., Zhanel, G.G., Schweizer, F. (2018). Antibiotic Hybrids: the Next Generation of Agents and Adjuvants against Gram-Negative Pathogens? Clin Microbiol Rev., 31(2):e0007717. doi: 10.1128/CMR.00077-17.

33. Yang, X., Ammeter, D., Idowu, T., Domalaon, R., Brizuela, M., Okunnu, O., Bi, L., Guerrero, Y.A., Zhanel, G.G., Kumar, A., Schweizer, F. (2019). Amphiphilic Nebramine-Based Hybrids Rescue Legacy Antibiotics From Intrinsic Resistance in Multidrug-Resistant Gram-negative Bacilli. Eur J Med Chem., 175:187-200. doi: 10.1016/j.ejmech.2019.05.003.

34. Martin-Serrano, A., Gomez, R., Ortega, P., de la Mata, F.J. (2019). Nanosystems as Vehicles for the Delivery of Antimicrobial Peptides (AMPs). Pharmaceutics, 11(9):448. doi: 10.3390/ pharmaceutics 11090448 .

35. Biswaro, L.S., da Costa Sousa, M.G., Rezende, T.M.B., Dias, S.C., Franco, O.L. (2018). Antimicrobial Peptides and Nanotechnology, Recent Advances and Challenges. Front Microbiol., 9:855. doi: 10.3389/fmicb.2018.00855.

36. Naskar, A., Kim, K. S. (2019). Nano-materials as Delivery Vehicles and Components of New Strategies to Combat Bacterial Infections: Advantages and Limitations. Microorganisms, 7(9):356. doi: 10.3390/microorganisms 7090356 .

37. Afrasiabi, S., Pourhajibagher, M., Raoofian, R., Tabarzad, M., Bahador, A. (2020). Therapeutic Applications of Nucleic Acid Aptamers in Microbial Infections. J Biomed Sci, 27 (1), 6. doi: 10.1186/ s12929-019-0611-0.

38. Ruddaraju, L.K., Pammi, S.V.N., Guntuku, G.S., Padavala, V.S., Kolapalli, V.R.M. (2020). A Review on Anti-Bacterials to Combat Resistance: From Ancient Era of Plants and Metals to Present and Future Perspectives of Green Nano Technological Combinations. Asian J Pharm Sci, 15 (1), 42-59. doi: 10.1016/j.ajps.2019.03.002.

39. Sultan, I., Rahman, S., Jan, A.T., Siddiqui, M.T., Mondal, A.H., Haq, Q.M.R. (2018). Antibiotics, Resistome and Resistance Mechanisms: A Bacterial Perspective. Front Microbiol., 9:2066. doi: 10.3389/ fmicb.2018.02066.

40. Wojciechowska, M., Rownicki, M., Mieczkowski, A., Miszkiewicz, J., Trylska, J. (2020). Antibacterial Peptide Nucleic Acids-Facts and Perspectives. Molecules. 25(3):559. doi: 10.3390/ molecules25030559.

41. Mulani, M. S., Kamble, E.E., Kumkar, S.N., Tawre, M. S., Pardesi, K.R. (2019). Emerging Strategies to Combat ESKAPE Pathogens in the Era of Antimicrobial Resistance: A Review. Front Microbiol. 10:539. doi: 10.3389/fmicb.2019.00539.

42. Liu, Y., Li, R., Xiao, X., Wang, Z. (2019). Antibiotic adjuvants: an alternative approach to overcome multi-drug resistant Gram-negative bacteria. Crit Rev Microbiol., 45(3):301-314. doi: 10.1080/ 1040841X.2019.1599813.

43. Marx, C., Gardner, S., Harman, R.M., Van de Walle, G.R. (2020). The mesenchymal stromal cell secretome impairs methicillin-resistant Staphylococcus aureus biofilms via cysteine protease activity in the equine model. Stem Cells Transl Med., 1-12. doi: 10.1002/sctm.19-0333. 
44. Gupta, V., Datta, P. (2019). Next-generation strategy for treating drug resistant bacteria: Antibiotic hybrids. Indian J Med Res. 2019 Feb; 149(2): 97-106. doi: 10.4103/ijmr.IJMR_755_18.

45. Heras, B., Scanlon, M.J., Martin, J.L. (2015). Targeting virulence not viability in the search for future antibacterials. Br J Clin Pharmacol. 79(2):208-15. doi: 10.1111/bcp.12356.

46. Gajd?cs, M., Spengler, G. (2019). The Role of Drug Repurposing in the Development of Novel Antimicrobial Drugs: Non-Antibiotic Pharmacological Agents as Quorum Sensing-Inhibitors. Antibiotics (Basel) 8(4):270. doi: 10.3390/antibiotics8040270.

47. Jansen, K.U., Anderson, A.S. (2018). The role of vaccines in fighting antimicrobial resistance (AMR). Hum Vaccin Immunother., 14(9):2142-2149. doi: 10.1080/21645515.2018.1476814.

48. Buchy, P., Ascioglu, S., Buisson, Y., Datta, S., Nissen, M., Tambyah, P.A., Vong, S. (2020). Impact of Vaccines on Antimicrobial Resistance. Int J Infect Dis., 90:188-196. doi: 10.1016/ j.ijid.2019.10.005.

49. Krishnamurthy, M., Moore, R.T., Rajamani, S., Panchal, R.G. (2016). Bacterial genome engineering and synthetic biology: combating pathogens. BMC Microbiol. 16(1):258. doi: 10.1186/ s12866-016-0876-3.

50. Duval, R. E., Grare, M., Demor?, B. (2019). Fight Against Antimicrobial Resistance: We Always Need New Antibacterials but for Right Bacteria. Molecules. 24(17):3152. doi: 10.3390/molecules24173152.

51. Bax, R., Green, S. (2015). Antibiotics: the changing regulatory and pharmaceutical industry paradigm. J Antimicrob Chemother. 70(5):1281-4. doi: 10.1093/jac/dku572.

Received: 23-Feb-2020

Accepted: 06-May-2020 